\title{
ON THE EXISTENCE OF A MIXING MEASURE
}

\author{
JOHN C. HOLLADAY ${ }^{1}$
}

Introduction. An interesting but apparently neglected generalization due to Doob [1], of the ergodic theorem, is the generalization to transformations which are not necessarily one-to-one. In his paper $[1]$, Doob showed that the transformation of the interval $[0,1]$ into itself given by $f(x)=n x$ (modulo 1 ), where $n-1$ is a positive integer, is ergodic under Lebesgue measure and that the law of large numbers is a trivial corollary of this ergodicity. This mapping is not only ergodic, but may be shown to be strongly mixing (which I shall just call mixing). Also, other similar transformations such as $f(x)$ $=2 x(0 \leqq x \leqq 1 / 2), f(x)=2-2 x(1 / 2 \leqq x \leqq 1)$ may similarly be shown to be mixing.

In a talk at Chicago on April 26, 1952, S. M. Ulam suggested that for sufficiently smooth transformations of a certain type, there might exist measures continuously related to Lebesgue measure under which such transformations are mixing. The main purpose of this paper is to show the validity of this speculation.

The statement in the theorem of the uniqueness of $F \in L_{1}$ which produces an invariant measure does not imply that there are no more invariant measures which are topologically equivalent to Lebesgue measure. In another paper, On conjugacy of some transformations of the interval, I shall show, among other things, that given any function of this type for which there is an invariant measure assigning every open sub-interval a positive measure, there exists an uncountable number of distinct measures, topologically equivalent to Lebesgue measure, under which the function is mixing. Also, there are many more such measures under which the transformation is invariant but not even ergodic.

Definition. (a) Let a transformation $f$ be called invariant under a measure $\mu$ if and only if given a measurable set $E, \mu(E)=\mu\{x / f(x) \in E\}$.

(b) Let a transformation $f$ be called ergodic under a measure $\mu$ if and only if given measurable sets $E$ and $F$,

$$
\mu(E) \mu(F)=\lim _{n \rightarrow \infty} \frac{1}{n} \sum_{i=1}^{n} \mu\left\{x \mid x \in E, f^{i}(x) \in F\right\} .
$$

(c) Let a transformation $f$ be called mixing under a measure $\mu$ if and only if given measurable sets $E$ and $F$,

Received by the editors September 25, 1956.

1 Work done under the auspices of the AEC. 


$$
\mu(E) \mu(F)=\lim _{n \rightarrow \infty} \mu\left\{x \mid x \in E, f^{n}(x) \in F\right\} .
$$

It is easily shown that mixing implies ergodicity which implies invariancy, and if $\mu$ is not the zero measure, ergodicity implies that the measure of the whole space is one. Also, we know that if $f$ is invariant and $\mu(E) \mu(F)>0$ implies that $\mu\left\{x \mid x \in E, f^{n}(x) \in F\right\}>0$ for some $n$, then $f$ is ergodic under $\mu / \mu$ (the space) [2]. Furthermore, if $\mu$ is not the zero measure, and $f$ is ergodic, $\mu(E)=\lim _{n \rightarrow \infty}(1 / n)$ [the number of the points $x, f(x), \cdots, f^{n}(x)$ which are contained in $E$ ] for almost all $x$ [2].

Since step functions are dense in $L_{1}(\mu)$ and $L_{\infty}(\mu)$, it is easily shown that:

(a) $f$ is invariant under $\mu$ if and only if

$$
\int \phi(x) \mu(d x)=\int \phi[f(x)] \mu(d x) \text { for all } \phi \in L_{1}(\mu) .
$$

(b) $f$ is ergodic under $\mu$ if and only if

$$
\left[\int \phi_{1}(x) \mu(d x)\right]\left[\int \phi_{2}(x) \mu(d x)\right]=\lim _{n \rightarrow \infty} \frac{1}{n} \sum_{i=1}^{n} \int \phi_{1}(x) \phi_{2}\left[f^{i}(x)\right] \mu(d x)
$$

for all $\phi_{1} \in L_{1}(\mu)$ and $\phi_{2} \in L_{\infty}(\mu)$.

(c) $f$ is mixing under $\mu$ if and only if

$$
\left[\int \phi_{1}(x) \mu(d x)\right]\left[\int \phi_{2}(x) \mu(d x)\right]=\lim _{n \rightarrow \infty} \int \phi_{1}(x) \phi_{2}\left[f^{n}(x)\right] \mu(d x),
$$

for all $\phi_{1} \in L_{1}(\mu)$ and $\phi_{2} \in L_{\infty}(\mu)$.

Definition. Let a transformation $f$ of $[0,1]$ into $[0,1]$ be called a zig-zag function if and only if there exists a finite set $P$ in $[0,1]$ containing the points 0 and 1 and at least one other point such that

(a) $f(P)$ is contained in $P$.

For each component $C$ of $[0,1]-P$,

(b) $f$ maps $C$ homeomorphically.

(c) The end points of $f(C)$ are points of $P$.

(d) For some positive integer $n, f^{n}(C)-P=[0,1]-P$.

Theorem. Let $f$ be a zig-zag function and let $d f(x) / d x$ exist and be uniformly continuous on each component of $[0,1]-P$. Also, let there exist a positive integer $p$ and a number $b_{p}>1$ so that for $f^{p-1}(x) \notin P$, $\left|d f^{p}(x) / d x\right|>b_{p}$.

Then there exists a unique function $F$ in $L_{1}$ (the space of Lebesgue integrable functions on $[0,1]$ ), such that 


$$
\int_{0}^{1} F(x) d x=1
$$

and $f$ is invariant under the measure $\mu$ given by $\mu(d x)=F(x) d x$. Furthermore, $f$ will be mixing under $\mu ; F$ will have a positive lower bound, and $F$ will be uniformly continuous on $[0,1]-P$.

Proof. In my paper [3], I prove the following theorem: Let $S$ be a compact Hausdorff space and $V^{+}$a nontrivial closed subset of the set of non-negative continuous functions on $S$. If $\lambda$ is a non-negative real number and $\phi \in V^{+}$, let $\lambda \phi \in V^{+}$. Also, let the set

$$
\left\{\phi \mid \phi \in V^{+},\|\phi\| \equiv \max _{s \in S} \phi(s)=1\right\}
$$

be equicontinuous. Let $T$ be a continuous mapping of $V^{+}$into $V^{+}$ such that

(a) If $\lambda$ is a non-negative real number, then $T(\lambda \phi)=\lambda T(\phi)$.

(b) If $\phi_{1}(s) \leqq \phi_{2}(s)$ for all $s \in S$, then $\left[T \phi_{1}\right](s) \leqq\left[T \phi_{2}\right](s)$ for all $s \in S$.

(c) If $\phi_{1}(s) \leqq \phi_{2}(s)$ for all $s \in S$, and $\phi_{1} \neq \phi_{2}$, then there exists a positive integer $n$ such that $\left[T^{n} \phi_{1}\right](s)<\left[T^{n} \phi_{2}\right](s)$ for all $s \in S$.

Then there exists a unique function $F \in V^{+}$such that $\|F\|=1$ and $T(F)=\lambda F$ for some real number $\lambda$. Furthermore, $F$ is positive valued and for any $\phi \in V^{+}$which is not the null function, $T^{i}(\phi) /\left\|T^{i}(\phi)\right\|$ converges to $F$ as the integer $i$ tends to infinity.

For our purposes, we are interested in non-negative valued functions $\phi$ on $[0,1]-P$ such that for each component $C$ of $[0,1]-P$, $\phi$ may be extended so as to be continuous on $\bar{C}$. Therefore, the set $S$ for the above theorem could be thought of as the discrete union of the closures of the components of $[0,1]-P$.

The transformation in which we are interested is the transformation $T$ such that for

$$
y \in[0,1]-P, \quad[T \phi](y)=\sum_{f(x)=y} \frac{\phi(x)}{\left|\frac{d}{d x} f(x)\right|} .
$$

It is easily seen that for $\phi_{1} \in L_{1}$ and $\phi_{2} \in L_{\infty}$,

$$
\int_{0}^{1} \phi_{1}(x) \phi_{2}[f(x)] d x=\int_{0}^{1}\left[T \phi_{1}\right](x) \phi_{2}(x) d x .
$$

That $T$ is a continuous mapping of $C(S)$ into $C(S)$, one need only observe the obvious fact that for $\phi_{1}$ and $\phi_{2}$ in $C(S)$, 


$$
\left\|T\left(\phi_{1}\right)-T\left(\phi_{2}\right)\right\| \leqq \| \phi_{1}-\phi_{2}|| \sup _{s \in[0,1]-P} \sum_{f(:=)=s} \frac{1}{\left|\frac{d}{d x} f(x)\right|}
$$

and that this supremum is finite.

Property (a) of the above quoted theorem is obviously true. In fact, $T$ is not only homogeneous but linear. Property (b) is also obvious. To show property (c), it is first necessary to show that given any nondegenerate interval $I$, there exists a positive integer $n(I)$ so that $f^{n}(I)-P=[0,1]-P$. But since $d f^{p}(x) / d x>b_{p}>1$ for $f^{p-1}(x) \notin P$, there exists a positive integer $i$ so that $f^{i p}(I)$ contains some component $C$ of $[0,1]-P$. Choose $n(C)$ so that $f^{n(C)}(C)-P=[0,1]-P$. Then $[0,1]-P \supset f^{n(C)+i p}(I)-P=f^{n(C)}\left[f^{i p}(I)\right]-P \supset f^{n(C)}(C)-P=[0,1]-P$. Therefore, set $n(I)=n(C)+i p$. To show property (c) for $T$, choose $\phi_{1}$ and $\phi_{2}$ so that $\phi_{1}(s) \leqq \phi_{2}(s)$ for all $s$ but $\phi_{1} \neq \phi_{2}$. Choose a nondegenerate interval $I$ so that $\phi_{2}(s)-\phi_{1}(s)$ has a positive lower bound for $s \in I$. Then for $s \in S$,

$$
\left[T^{n(I)} \phi_{1}\right](s)<\left[T^{n(I)} \phi_{2}\right](s) .
$$

For $j$ a positive integer, we have that

$$
\begin{aligned}
{\left[T^{i} \phi\right](y) } & =\sum_{f^{j}(x)=y} \phi(x) /\left|\prod_{i=0}^{j-1} \frac{d}{d f^{i}(x)} f^{i+1}(x)\right| \\
& \equiv \sum_{f^{j}(x)=y} \phi(x) /\left|\frac{d}{d x} f^{j}(x)\right| .
\end{aligned}
$$

Since $d f(x) / d x$ is uniformly continuous for $x \in P$ and $\left|d f^{p}(x) / d x\right|$ has a positive lower bound $b_{p}$ for $f^{p-1}(x) \notin P$, it follows that $\left|d f^{i}(x) / d x\right|$ is uniformly continuous and has a positive lower bound $b_{j}$ for $f^{j-1}(x) \notin P$. For $\epsilon$ a positive number, define $m_{j}(\epsilon)$ as the supremum of $\left|d f^{i}\left(x_{1}\right) / d x-d f^{i}\left(x_{2}\right) / d x\right|$ for $\left|x_{1}-x_{2}\right| \leqq \epsilon$ and $f^{i}\left(x_{1}\right)$ in the same component of $[0,1]-P$ as $f^{i}\left(x_{2}\right),(0 \leqq i<j)$. Choose $\epsilon_{0}>0$ so that $\left[1+m_{p}\left(\epsilon_{0} / b_{p}\right)\right] / b_{p}$, call it $k$, is less than one. Choose a monotonic positive valued function $M$ on $\left(0, \epsilon_{0}\right]$ so that $M(\epsilon)>m_{p}(\epsilon) / b_{p}(1-k)$ and $M(\epsilon)$ approaches zero as $\epsilon$ tends to zero. Now define $A$ as $\{\phi \mid \phi(s)>0$ for $s \in S$; if $x_{1}$ and $x_{2}$ are in the same component of $[0,1]-P$ and $0<\left|x_{1}-x_{2}\right| \leqq \epsilon_{0}$, then $\left.\left|\left(\phi\left(x_{1}\right)-\phi\left(x_{2}\right)\right) / \phi\left(x_{1}\right)\right| \leqq M(\epsilon)\right\}$. Define the set $V^{+}$as the set of all functions which is contained in one of the sets $\bar{A}, T(\bar{A}), T^{2}(\bar{A}), \cdots, T^{p-1}(\bar{A})$. Note that since $T$ maps positive valued functions into positive valued functions, the only function in $V^{+}$ that is not positive valued is the identically zero-valued function.

Let $\phi$ be a function in $A$ and let $0<j \leqq p$. Let $x_{1}$ and $x_{2}$ be such 
that $f^{i}\left(x_{1}\right)$ is in the same component of $[0,1]-P$ as $f^{i}\left(x_{2}\right)$ for $0 \leqq i \leqq j$ and such that $0<\left|x_{1}-x_{2}\right| \leqq \epsilon \leqq \epsilon_{0}$. Then

$$
\begin{aligned}
&\left|\frac{1}{\phi\left(x_{1}\right) / \frac{d}{d x} f^{j}\left(x_{1}\right)}\left[\frac{\phi\left(x_{1}\right)}{\frac{d}{d x} f^{j}\left(x_{1}\right)}-\frac{\phi\left(x_{2}\right)}{\frac{d}{d x} f^{i}\left(x_{2}\right)}\right]\right| \\
&=\left|\frac{\frac{d}{d x} f^{i}\left(x_{1}\right)}{\frac{d}{d x} f^{j}\left(x_{2}\right)} \frac{\phi\left(x_{1}\right)-\phi\left(x_{2}\right)}{\phi\left(x_{1}\right)}-\frac{\frac{d}{d x} f^{j}\left(x_{1}\right)-\frac{d}{d x} f^{j}\left(x_{2}\right)}{\frac{d}{d x} f^{i}\left(x_{2}\right)}\right| \\
& \leqq \frac{1+m_{j}(\epsilon)}{b_{j}} M(\epsilon)+\frac{m_{j}(\epsilon)}{b_{j}} .
\end{aligned}
$$

Also by the law of the mean, $\left|x_{1}-x_{2}\right| \leqq\left|f^{j}\left(x_{1}\right)-f^{j}\left(x_{2}\right)\right| / b_{j}$. Consequently if $\phi \in A$, then for $\left|y_{1}-y_{2}\right| \leqq b_{j} \epsilon_{0}$ and $y_{1}$ and $y_{2}$ in the same component of $[0,1]-P$, by observing the above inequality for all appropriate pairs $x_{1}$ and $x_{2}$ mapping into $y_{1}$ and $y_{2}$, we see that

$$
\begin{aligned}
\left|\frac{\left[T^{i} \phi\right]\left(y_{1}\right)-\left[T^{i} \phi\right]\left(y_{2}\right)}{\left[T^{i} \phi\right]\left(y_{1}\right)}\right| \leqq & \frac{1+m_{j}\left(\frac{\left|y_{1}-y_{2}\right|}{b_{j}}\right)}{b_{j}} M\left(\frac{\left|y_{1}-y_{2}\right|}{b_{j}}\right) \\
& +\frac{m_{j}\left(\frac{\left|y_{1}-y_{2}\right|}{b_{j}}\right)}{b_{j}} .
\end{aligned}
$$

Consequently, for all positive $\phi \in V^{+}|\Delta \phi / \phi|$ is uniformly "well behaved" so that $\left\{\phi \mid \phi \in V^{+},\|\phi\|=1\right\}$ is equicontinuous.

To prove that $T$ maps $V^{+}$into itself, it is obviously sufficient to prove that $T^{p}$ maps $A$ into $A$. If $\phi \in A$ and $\left|y_{1}-y_{2}\right| \leqq \epsilon \leqq \epsilon_{0}$ and $y_{1}$ and $y_{2}$ are in the same component of $[0,1]-P$, then applying the above inequality for $j=p$,

$$
\begin{aligned}
& \left|\frac{\left[T^{p} \phi\right]\left(y_{1}\right)-\left[T^{p} \phi\right]\left(y_{2}\right)}{\left[T^{p} \phi\right]\left(y_{1}\right)}\right| \leqq \frac{1+m_{p}\left(\epsilon / b_{p}\right)}{b_{p}} M\left(\epsilon / b_{p}\right)+\frac{m_{p}\left(\epsilon / b_{p}\right)}{b_{p}} \\
& \quad \leqq \frac{1+m_{p}\left(\epsilon_{0} / b_{p}\right)}{b_{p}} M(\epsilon)+\frac{m_{p}(\epsilon)}{b_{p}}=k M(\epsilon)+\frac{m_{p}(\epsilon)}{b_{p}} \\
& \quad=k M(\epsilon)+(1-k) \frac{m_{p}(\epsilon)}{b_{p}(1-k)} \leqq k M(\epsilon)+(1-k) M(\epsilon)=M(\epsilon) .
\end{aligned}
$$


Therefore, $T^{p}(\phi)$ is in $A$ if $\phi$ is in $A$.

Now applying my above mentioned theorem to this transformation (and considering the $L_{1}$ norm instead of the continuous function norm), we see that there exists a unique $F$ in $V^{+}$such that $\int_{0}^{1} F(x) d x$ $=1$ and $T(F)=\lambda F$ for some real $\lambda$. Since $F \in V^{+}, F$ has a positive lower bound and is uniformly continuous on $[0,1]-P$. If we set $\phi_{1}$ $=F$ and $\phi_{2} \equiv 1$ and apply $\left({ }^{*}\right), 1=\int_{0}^{1} F(x) d x=\int_{0}^{1}[T F](x) d x=\int_{0}^{1} \lambda F(x) d x$ $=\lambda$. Therefore, $T(F)=F$.

Given any function $\phi \in L_{1}, \phi$ may be expressed as $\phi_{+}-\phi_{-}$where both $\phi_{+}$and $\phi_{-}$are non-negative functions so that $\|\phi\|_{1}=\left\|\phi_{+}\right\|_{1}$ $+\left\|\phi_{-}\right\|_{1}$. Therefore, using $\left(^{*}\right)$ with $\phi_{2} \equiv 1,\|T(\phi)\|_{1}=\left\|T\left(\phi_{+}\right)-T\left(\phi_{-}\right)\right\|_{1}$ $\leqq\left\|T\left(\phi_{+}\right)\right\|_{1}+\left\|T\left(\phi_{-}\right)\right\|_{1}=\int_{0}^{1}\left[T \phi_{+}\right](x) d x+\int_{0}^{1}\left[T \phi_{-}\right](x) d x=\int_{0}^{1} \phi_{+}(x) d x$ $+\int_{0}^{1} \phi_{-}(x) d x=\|\phi\|_{1}$. Repeating this argument, if $n$ is a positive integer $\left\|T^{n}(\phi)\right\|_{1} \leqq\|\phi\|_{1}$.

Repeatedly applying (*) with $\phi_{2} \equiv 1$, if $\phi=\phi_{1} \in V^{+}$, then

$$
T^{n}(\phi) / \int_{0}^{1} \phi(x) d x=T^{n}(\phi) / \int_{0}^{1}\left[T^{n}(\phi)\right](x) d x
$$

which, applying the previously mentioned theorem, converges to $F$ as $n$ tends to infinity. Therefore $T^{n}(\phi)$ converges to $F \int_{0}^{1} \phi(x) d x$ for $\phi \in V^{+}$. Consider a function $\phi \in L_{1}$ and choose $\epsilon>0$. Choose $\phi_{L}$ and $\phi_{\delta}$ so that $\phi_{L}$ is a finite linear combination of functions in $V^{+}$and $\left\|\phi_{\delta}\right\|_{1}<\epsilon / 3$ and $\phi=\phi_{L}+\phi_{\delta}$. Then

$$
\begin{aligned}
\| T^{n}(\phi)- & F \int_{0}^{1} \phi(x) d x\left\|_{1} \leqq\right\| T^{n}\left(\phi_{L}\right)-F \int_{0}^{1} \phi_{L}(x) d x\left\|_{1}+\right\| T^{n}\left(\phi_{\delta}\right) \|_{1} \\
& +\left\|F \int_{0}^{1} \phi_{\delta}(x) d x\right\|_{1}<\left\|T^{n}\left(\phi_{L}\right)-F \int_{0}^{1} \phi_{L}(x) d x\right\|_{1}+\epsilon / 3+\epsilon / 3
\end{aligned}
$$

which for $n$ a sufficiently large integer is less than $\epsilon$.

If $\phi_{1} \in L_{1}$ (and therefore $\phi_{1} F \in L_{1}$ ) and $\phi_{2} \in L_{\infty}$, then

$$
\int_{0}^{1} \phi_{1}(x) \phi_{2}\left[f^{n}(x)\right] F(x) d x=\int_{0}^{1}\left[T^{n}\left(\phi_{1} F\right)\right](x) \phi_{2}(x) d x,
$$

which, as $n$ tends to infinity, approaches

$$
\begin{aligned}
\int_{0}^{1}\left[\int_{0}^{1} \phi_{1}(x) F(x) d x\right] F(x) \phi_{2}(x) d x & \\
= & {\left[\int_{0}^{1} \phi_{1}(x) F(x) d x\right]\left[\int_{0}^{1} \phi_{2}(x) F(x) d x\right] . }
\end{aligned}
$$


Therefore, the measure $\mu$ obtained from $\mu(d x)=F(x) d x$ is mixing which implies that $\mu$ is invariant.

Let $F^{*}$ be a function in $L_{1}$ so that $\int_{0}^{1} F^{*}(x) d x=1$ and $f$ is invariant under the measure $\mu^{*}$ given by $\mu^{*}(d x)=F^{*}(x) d x$. Then for $\phi \in L_{\infty}$ and $n$ a positive integer, $\int_{0}^{1} \phi(x) F^{*}(x) d x=\int_{0}^{1} \phi\left[f^{n}(x)\right] F^{*}(x) d x$ $=\int_{0}^{1} \phi(x)\left[T^{n}\left(F^{*}\right)\right](x) d x$ which, as $n$ tends to infinity, approaches $\int_{0}^{1} \phi(x)\left[\int_{0}^{1} F^{*}(x) d x\right] F(x) d x=\int_{0}^{1} \phi(x) F(x) d x$. Since $\int_{0}^{1} \phi(x) F^{*}(x) d x$ $=\int_{0}^{1} \phi(x) F(x) d x$ for all $\phi \in L_{\infty}, F^{*}=F$ almost everywhere.

\section{BIBLIOGRAPHY}

1. J. L. Doob, The law of large numbers for continuous stochastic processes, Duke Math. J. vol. 6 (1940) pp. 290-306.

2. F. Riesz, Sur la théorie ergodique, Comment. Math. Helv. vol. 17 (1945) pp. 221239.

3. J. C. Holladay, On convergence to eigenfunctions for iterates of homogeneous transformations, Proc. Amer. Math. Soc. to be published.

Los Alamos Scientific Laboratory 